\title{
A Solution-processed Inorganic Emitter with High Spectral Effectiveness for Efficient Daytime Radiative Cooling in Hot Humid Climates
}

\author{
Chongjia Lin ( $\nabla$ clinag@connect.ust.hk) \\ Hong Kong University of Science and Technology \\ Yang Li \\ Hong Kong University of Science and Technology \\ Cheng Chi \\ Tsinghua University \\ Ye Seul Kwon \\ The Hong Kong University of Science and Technology \\ Chi Yan Tso \\ City University of Hong Kong \\ Christopher Chao \\ University of Hong Kong

\section{Baoling Huang} \\ The Hong Kong University of Science and Technology
}

\section{Article}

Keywords: Daytime radiative cooling, all-inorganic narrowband cooler, solution-based fabrication

Posted Date: May 24th, 2021

DOI: https://doi.org/10.21203/rs.3.rs-235967/v1

License: @ (i) This work is licensed under a Creative Commons Attribution 4.0 International License. Read Full License 


\section{Abstract}

Daytime radiative cooling provides an eco-friendly solution to space cooling with zero energy consumption. Despite significant advances, most state-of-the-art radiative coolers show broadband infrared emission with low spectral effectiveness, which limits their cooling temperatures and climate applicabilities, especially in hot humid regions. Here we report an all-inorganic narrowband cooler comprising a solution-derived $\mathrm{SiO}_{x} \mathrm{~N}_{y}$ layer sandwiched between a reflective substrate and a self-assembly monolayer of $\mathrm{SiO}_{2}$ microspheres. It shows a high and diffusive solar reflectance (96\%) and strong infrared-selective emittance (94.6\%) with superior spectral effectiveness (1.44). Remarkable subambient cooling of up to $5^{\circ} \mathrm{C}$ was achieved under high humidity without any solar shading or convection cover at noontime in a subtropical coastal city, Hong Kong. Owing to the all-inorganic hydrophobic structure, the emitter showed outstanding resistance to ultraviolet and water in the long-term durability tests. The scalable solution-based fabrication renders this stable high-performance emitter promising for large-scale deployment in various climates.

\section{Introduction}

Passive radiative cooling, in which heat is spontaneously radiated by surfaces to the higher sky with a low temperature through the atmospheric infrared (IR) windows, provides a sustainable solution to space cooling with zero energy consumption ${ }^{1,2}$. Although nighttime cooling has been utilized for centuries, it was only recently that daytime cooling was first demonstrated using a multilayer photonic structure ${ }^{3}$. In tropical and subtropical areas, the demand for cooling is particularly huge due to high ambient temperatures. However, it has proved difficult to achieve daytime cooling in such areas with stronger solar irradiation and higher humidity than midlatitude regions $\mathrm{s}^{4-8}$. With the increase in humidity, the transmittances within the atmospheric windows remarkably decrease while some such as the 16-25 um window may even vanish in humid climates (Fig. 1a, Supplementary Fig. S1). This significantly increases the atmospheric radiation and limits the cooling capability of radiative coolers. To maintain applicability in different climates, an ideal daytime radiative cooler should show high emittance only within the main 8-13 $\mu \mathrm{m}$ atmospheric window $\left(\varepsilon_{8-13 \mu \mathrm{m}}\right)$ to maximize emission power, but high reflectance $R$ beyond this window to minimize the received radiation from the sun and atmosphere, i.e., a narrowband IR emission with high spectral effectiveness $\mathbb{B}_{\varepsilon}=\varepsilon_{8-13 \mu \mathrm{m}} / \varepsilon_{0-\infty \mu \mathrm{m}}{ }^{3,9-11}$.

Over the past few years, significant efforts have been made to improve the daytime radiative cooling performance and reduce the costs. ${ }^{11-15}$ The first reported daytime emitter was a multilayer photonic crystal ${ }^{3}$, showing a high $R_{\text {solar }}=97 \%$, reasonable $\varepsilon_{8-13 \mu m}=65 \%$ and a high $\mathbb{B}_{\varepsilon}=1.48$. Later on, other multilayer emitters ${ }^{16,10}$ with improved $\varepsilon_{8-13 \mu m}$ were developed, but their large-scale deployment was restricted by high fabrication costs. Thus, scalable designs adopting dispersed inorganic particles ${ }^{17,18,19}$, porous polymers ${ }^{20}$ and even woods ${ }^{21}$ were developed to achieve both high $R_{\text {solar }}(>94 \%)$ and high $\varepsilon_{8-13 \mu m}(>90 \%)$. These low-cost emitters enable the practical applications of daytime radiative cooling, but they normally show broadband IR emission and low $\mathbb{E}_{\varepsilon}(\sim 1)$. Meanwhile, they often adopt polymer matrices and the aging issue caused by long-time exposure to ultraviolet and moisture is a concern ${ }^{22}$. Hence, it is urgently demanded to develop scalable IR-selective radiative coolers with great long-term stability in the harsh outdoor environment.

Here we demonstrate a robust all-inorganic narrowband emitter with strong diffusive solar reflection $(R=96 \%)$ and highly selective IR emission $\left(\varepsilon_{8-13 \mu \mathrm{m}}=94.6 \%\right)$, which can be fabricated by scalable solution processes at room temperature. Its high IR emittance mainly locates within the major atmospheric window, leading to a high spectral effectiveness $\mathbb{V}_{\varepsilon}=1.44$, which is the highest among state-of-theart emitters with comparable $\varepsilon_{8-13 \mu \mathrm{m}}$. Temperature drops of $5^{\circ} \mathrm{C}$ were achieved in a subtropical coastal city, Hong Kong, with the emitter directly exposed to sunlight at noontime $(\mathrm{RH}=\sim 50 \%)$ without adopting any solar shading structure or convection cover. Such cooling temperatures could be maintained even when the relative humidity increased to $90 \%$ at nighttime. Moreover, the inorganic cooler exhibited exceptional long-term stability after ultraviolet exposure (3 months) and water immersion ( 3 weeks), which is critical for practical deployments.

\section{Results And Discussion}

The sphereenhanced emitter and its spectrum. The emitter has a triple-layer structure, comprising a monolayer of closely packed silica microspheres, a silicon oxynitride $\left(\mathrm{SiO}_{x} \mathrm{~N}_{y}\right)$ layer derived from perhydropolysilazane (PHPS) and a silver layer on a substrate (Figs. 1b-c). This simple all-inorganic design can generate highly selective but strong IR emission (Fig. 1a) by rationally exploiting the optical behaviors of the $\mathrm{SiO}_{\mathrm{x}} \mathrm{N}_{\mathrm{y}}$ layer and silica microspheres. 
The emitter was fabricated using facile solution processes (Fig. 1e). PHPS, an inorganic precursor containing Si-N skeleton, was used to generate a dense $\mathrm{SiO}_{x} \mathrm{~N}_{y}$ layer on a silver-coated substrate through natural solidification in the air (Supplementary Fig. S2-4). The $\mathrm{SiO}_{\mathrm{x}} \mathrm{N}_{\mathrm{y}} / \mathrm{Ag}$ structure was mirror-like and showed highly selective IR emission $\left(\varepsilon_{8-13 \mu \mathrm{m}} \approx 80 \%\right.$ and $\left.\mathbb{Z}_{\varepsilon}>1.4\right)$, as shown in Fig. $1 \mathrm{a}$. Another emissive peak caused by Si-H bonds ${ }^{23}$ was observed at $4.63 \mu \mathrm{m}$, which matches the $4.5-5 \mu \mathrm{m}$ atmospheric window. To enhance its IR emissivity while maintaining the spectral selectivity, a monolayer of $\mathrm{SiO}_{2}$ microspheres with diameters of $\sim 2 \mu \mathrm{m}$ was coated on top (Supplementary Fig. S5) using the Langmuir-Blodgett (LB) self-assembly process ${ }^{24}$, which has been widely used for roll-to-roll microparticle deposition. After immobilizing the silica microspheres with a thin PHPS-derived bonding layer, a white paper-like inorganic emitter was attained. Figure $1 \mathrm{~d}$ shows a $20 \mathrm{~cm} \times 20 \mathrm{~cm}$ sample fabricated on a silver-coated aluminum film.

The optical function of each component of the emitter is schematically shown in Fig. 2a. The $\mathrm{SiO}_{x} \mathrm{~N}_{\mathrm{y}}$ layer is transparent to sunlight but it generates intrinsic selective emission within the 8-13 $\mu \mathrm{m}$ atmospheric window. The monolayer of close-packed $\mathrm{SiO}_{2}$ microspheres above the $\mathrm{SiO}_{\mathrm{x}} \mathrm{N}_{\mathrm{y}}$ layer further selectively enhances the emissivity at the wavelengths near $9 \mu \mathrm{m}$ and $12 \mu \mathrm{m}$. The normalized emissive power of each part is shown in Supplementary Fig. S6. The bottom silver layer can elongate the optical path to improve the emittance within 8-13 $\mu \mathrm{m}$ and produce high reflection beyond the window. The coupling of these three layers leads to strong narrowband IR emission in the atmospheric window $\left(\varepsilon_{8-13 \mu m}=94.6 \%\right)$ and excellent spectral effectiveness $\left(\mathbb{B}_{\varepsilon}=1.44\right)$. Also, strong scatterings of visible light by the microspheres enable the transition from specular to diffusive reflection on the emitter surface (Fig. 1d). It is important in practical applications to avoid glare appearance and light pollution problems.

The refractive index of the PHPS-derived $\mathrm{SiO}_{x} \mathrm{~N}_{y}$ is shown in Fig. $2 \mathrm{~d}$ (upper panel). Owing to the Si-N and Si-O bonds ${ }^{23}$, its extinction

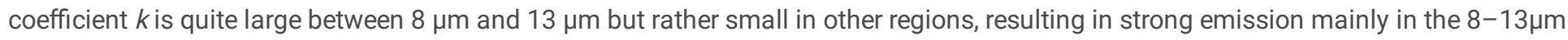
window. Figure $2 \mathrm{e}$ (upper panel) illustrates the emissivities of $\mathrm{SiO}_{x} \mathrm{~N}_{y}$ layers with different thicknesses on a reflective surface. Within the atmospheric window, its $\varepsilon_{8-13 \mu \mathrm{m}}$ rapidly rises to around $80 \%$ when the thickness increases to $2 \mu \mathrm{m}$, and then only slightly improves with the increasing thicknesses. Beyond the atmospheric window, the average emissivity keeps increasing with the increasing layer thickness because of the non-zero $k$ value. The highest effectiveness $\left(\mathbb{B}_{\varepsilon}=2.2\right)$ is achieved at a thickness of $\sim 0.6 \mu \mathrm{m}$ but the corresponding $\varepsilon_{8-13 \mu \mathrm{m}}$ is small; further increasing the thickness will gradually reduce the effectiveness to $\sim 1.1$ (Supplementary Fig. S7). Thus, if the layer thickness is controlled between 1.5 and $4 \mu \mathrm{m}$, the PHPS-derived emitter can offer both a high emissivity $\left(\varepsilon_{8-13 \mu m}>80 \%\right)$ and high effectiveness $\left(\mathbb{E}_{\varepsilon}>1.4\right)$. This large thickness tolerance provides great opportunities for cost reduction in large-scale fabrication, compared to previous photonic or plasmonic structures with nanometer-level tolerance.

The $\varepsilon_{8-13 \mu \mathrm{m}}$ of a single $\mathrm{SiO}_{x} \mathrm{~N}_{y}$ layer can reach $\sim 80 \%$, but it is difficult to be further improved without sacrificing the effectiveness $\mathbb{Z}_{\varepsilon}$ through increasing the thickness, because of the reflection peaks at around 9 and $12 \mu \mathrm{m}$ caused by the Reststrahlen bands of bulk $\mathrm{SiO}_{x} \mathrm{~N}_{y}$. Figure $2 \mathrm{e}$ (upper panel) clearly shows weaker emission near 9 and $12 \mu \mathrm{m}$ due to the Reststrahlen bands for almost all the thicknesses. Different from its bulk counterpart, $\mathrm{SiO}_{2}$ microspheres show resonance-enhanced light-matter interactions especially at wavelengths of around 9 and $12 \mu \mathrm{m}$ and therefore high absorption/emission ${ }^{19}$, which is perfectly complementary to the $\mathrm{SiO}_{\mathrm{x}} \mathrm{N}_{\mathrm{y}}$ layer (Fig. 2e (lower panel)). The resonance with strong near-field confinement was identified as surface phonon polaritons (SPhP). Further, the high-intensity near-field effect can be outcoupled into far-field through diffraction by a periodic surface structure and induce emission enhancement ${ }^{25}$. A close-packed monolayer can generate $20 \%$ more IR emission than that with randomly distributed spheres of the same $\mathrm{size}^{26}$. Compared with non-close-packed $\mathrm{SiO}_{2}$ microsphere monolayers of different periodicities, a close-packed monolayer shows the strongest resonance absorption (Supplementary Fig. S8). Meanwhile, increasing the number of microsphere layers rapidly broadens the emission spectrum. Therefore, a close-packed monolayer of $\mathrm{SiO}_{2}$ microspheres was deposited on top of $\mathrm{SiO}_{x} \mathrm{~N}_{y}$ to selectively enhance $\varepsilon_{8-13 \mu m}$.

The IR selectivity of $\mathrm{SiO}_{2}$ microspheres strongly relies on their size. Figure 2e (lower panel) shows the emissive spectra of a monolayer of $\mathrm{SiO}_{2}$ microspheres with different diameters on a reflective surface. When the diameter is larger than $1.6 \mu \mathrm{m}$, two strong narrowband emission peaks appear at desired wavelengths ( $9 \mu \mathrm{m}$ and $12 \mu \mathrm{m})$. When the size exceeds $2.6 \mu \mathrm{m}$, higher-order Fröhlich resonances will be excited beyond the Reststrahlen bands ${ }^{17}$, deteriorating the infrared selectivity. Hence, $\mathrm{SiO}_{2}$ spheres with sizes of $1.6-2.6 \mu \mathrm{m}$ are selected to provide complementary emission while keeping the IR selectivity. This is different from previous works using $8-\mu \mathrm{m} \mathrm{SiO}_{2}$ microspheres to realize broadband IR emssion ${ }^{17,26}$. Figures $2 \mathrm{~b}$-c show that the electric field at $\lambda=9 \mu \mathrm{m}$ is enhanced by seven-fold while that at $\lambda=12 \mu \mathrm{m}$ becomes 5 -fold stronger. With the complementary coupling of SPhP with the selective emission spectrum of the $\mathrm{SiO}_{x} \mathrm{~N}_{\mathrm{y}}$ layer, the microsphere-enhanced emitter shows a much-improved IR emissivity $\left(\varepsilon_{8-13 \mu \mathrm{m}}=94.6 \%\right)$ while maintaining a high $\mathbb{E}_{\varepsilon}$ of 1.44 
(Fig. 1a). The measured angular emissivity of the microsphere-enhanced emitter can maintain above $90 \%$ up to $60^{\circ}$ emissive angle (Supplementary Fig. S9).

When PHPS is annealed at low temperatures $\left(<300^{\circ} \mathrm{C}\right)$, the film is slightly hydrophobic due to low dispersive and polar forces ${ }^{23}$. The contact angle of the PHPS-derived $\mathrm{SiO}_{x} \mathrm{~N}_{y}$ layer near room temperature is close to $90^{\circ}$ (Fig. 2f). However, after depositing a monolayer of hydrophobic microspheres, the surface became more hydrophobic and the water contact angle reached $\sim 130^{\circ}$ (Fig. $2 \mathrm{~g}$ ). Such hydrophobic surfaces endow the emitters with water-repelling behaviours and self-cleaning capability, which may benefit their operation and maintenance in harsh outdoor environments.

The outdoor experiment in a subtropical coastal city (Hong Kong). The field test was conducted for 4 consecutive days (4-7 November 2019) on the roof of a seaside building in Hong Kong (Supplementary Fig. S10), where the daily averaged relative humidity ranges from $60-80 \%$ all over the year ${ }^{27}$. The experimental setup is shown in Figs. 3a-b. A 4-inch emitter was directly mounted on the top of a cubic high-density expanded polystyrene (EPS) foam without adopting any solar shading or convection cover (the so-called roof cooling mode). The EPS foam shows a spectrum very similar to those of commercial heat-reflective paints ( $R=92 \%$, Supplementary Fig. S11) and its thermal conductivity is only $0.035 \mathrm{~W} / \mathrm{m} \cdot \mathrm{k}^{28}$, which can minimize the absorbed heat transferring from the foam to the emitter. Type-T thermocouples were attached to the backside of the emitter via a small tunnel penetrating through the foam. To accurately measure the ambient air temperature, the heating from the concrete ground and the direct exposure of the thermometer under sunlight should be avoided, which might cause overestimation of the ambient air temperature and in turn the cooling temperature by at least 2$3^{\circ} \mathrm{C}$ according to our tests. Here we used a well-calibrated commercial weather station standing beside with a shutter box kept at the same height level as the emitter (about 1 meter from the floor) to accurately measure the ambient air temperature, relative humidity, solar irradiation and wind speed simultaneously

During the test, the sky was clear in the first 1.5 days but became cloudy thereafter (the cloud coverages were $47 \%$ and $32 \%$ respectively in the last two days according to the Hong Kong Observatory). The measured temperatures of ambient air and the emitters (including PHPS-derived and sphere-enhanced emitters) together with the solar irradiation are shown in Fig. $3 \mathrm{~d}$ while the measured wind speed and relative humidity are shown in Fig. 3e. The daytime cooling effects on clear days were obviously larger than on cloudy days while the emitter with microspheres consistently showed lower temperature than that without microspheres. For the sphere-enhanced emitter, about $5^{\circ} \mathrm{C}$ temperature drop below the ambient temperature was observed at noontime under $800 \mathrm{~W} / \mathrm{m}^{2}$ solar irradiation and $50 \% \mathrm{RH}$. This emitter maintained a cooling temperature of $3-5^{\circ} \mathrm{C}$ during the daytime even when the wind speed reached up to $2 \mathrm{~m} / \mathrm{s}$. When the cloud coverage increased to $~ 50 \%$, the cooling temperature decreased to $1-2^{\circ} \mathrm{C}$ at noontime. In the nighttime, the relative humidity increased rapidly to $80-90 \%$, but the temperature drop could still be maintained at $3-6^{\circ} \mathrm{C}$ with clear skies and $1-4^{\circ} \mathrm{C}$ with cloudy skies. Around $50 \mathrm{~W} / \mathrm{m}^{2}$ of cooling power was recorded on a clear night with a humidity level of around $70 \%$ (Fig. 3c). The corresponding nonradiative heat transfer coefficient was calculated to be $\sim 10 \mathrm{~W} / \mathrm{m}^{2} \mathrm{~K}$, agreeing well with the empirical formula attained by Zhao et $\mathrm{al}^{29}$.

Comparative study of roof cooling was also conducted using two wooden model houses $(60 \times 70 \times 85 \mathrm{~cm})$ with a standard thermal insulating roof and an emitter-assembled roof, respectively. Figure $3 \mathrm{f}$ shows the IR image of the houses at noontime (the ambient temperature was $\sim 31^{\circ} \mathrm{C}$ ). Due to the variation in surface orientation and side wall heating by sunlight, the emitter roof temperature varied with position and the lowest temperature could reach $24^{\circ} \mathrm{C}$. In contrast, the temperature of the standard roof ranged from $55^{\circ} \mathrm{C}$ to $67^{\circ} \mathrm{C}$. The average air temperature inside the house with the cooling roof was $\sim 8^{\circ} \mathrm{C}$ and $2^{\circ} \mathrm{C}$ lower than that of the reference house at daytime and nighttime, respectively (Supplementary Fig. S12). Besides, a 20×20 cm emitter was mounted on top of a 200-ml water container to cool water. Subambient water coolings of up to $7^{\circ} \mathrm{C}$ and $3^{\circ} \mathrm{C}$ were observed at nighttime and noontime, respectively (Supplementary Fig. S13). These tests show the great potential of this emitter for space cooling.

Cooling performance and durability evaluation of the emitters. Most emitters in the literature show high solar reflectances ( $>94 \%)$, but their infrared emissive spectra are quite different. Two indexes have been proposed to quantify the selectivity and cooling capability of a radiative surface ${ }^{29}$. One is the weighted emissivity $\varepsilon_{8-13 \mu \mathrm{m}}$ over the $8-13 \mu \mathrm{m}$ window at $T_{\mathrm{amb}}=300 \mathrm{~K}$ and the other is the spectral effectiveness $\mathbb{E}$. Since the secondary atmospheric windows only slightly contribute to the cooling performance, $\varepsilon_{8-13 \mu m}$ can indicate the emission power of an emitter and $\mathbb{\varepsilon \varepsilon}$ indicates the ratio of the surface emission to the absorption from the ambient, i.e., the capability in achieving a high cooling temperature in different climates $^{9,30}$.

The $\varepsilon_{8-13 \mu m}$ and $\varangle \varepsilon$ of various emitters for daytime cooling $3,16-18,20,21,31-38$ were calculated based on their spectra and plotted in Fig. 4a. Most emitters with a $\varepsilon_{8-13 \mu m}$ above $90 \%$ show a relatively low effectiveness $\mathbb{\Xi}_{\varepsilon}(<1.1)$, indicating their broadband emitter nature. 
So far, the highest $\nabla_{\varepsilon}(1.48)$ was achieved by the photonic emitter developed by Raman et. al ${ }^{3}$, which showed a $\varepsilon_{8-13 \mu m}$ of $65 \%$. Our emitter exhibits strong solar reflection $(96 \%)$, high IR emission $\left(\varepsilon_{8-13 \mu \mathrm{m}}=94.6 \%\right)$ and outstanding selectivity $(\varangle \varepsilon=1.44)$, showing a performance closest to an ideal selective emitter compared to state-of-the-art emitters (Fig. 4a). The cooling performance of the emitter in six typical climates from MODTRAN 6 was evaluated and compared with those of an ideal selective emitter and ideal IR-broadband emitter (Supplementary Fig. S14). Our emitter's behavior is close to that of the ideal selective emitter and can achieve a much larger cooling temperature than the ideal broadband emitter in different climates, illustrating its excellent cooling performance and climate applicability

The durability of daytime radiative emitters is crucial for long-term operation. UV exposure by sunlight and water immersion by rain are two main challenges for maintaining emitters' cooling performances in practical applications. UV can break some chemical bonds in organic materials and in turn modify the spectrum, causing degradation and aging problems ${ }^{22}$. Moisture and oxygen can attack the reflective layer through pinholes and induce matrix variation, while some materials with pores will absorb water and affect their optical properties $^{29}$. The dense all-inorganic structure of our emitter inherently endows it with excellent resistance to UV and water. The Si-O bond energy is larger than the energy of the shortest UV in sunlight ( $300 \mathrm{~nm}$ ) and water cannot pass through or accumulate in the dense hydrophobic $\mathrm{SiO}_{x} \mathrm{~N}_{y}$ / silica microsphere structure. To confirm this, we conducted durability tests with the emitter directly exposed to UV and water. A sphere-enhanced emitter was exposed in solar UV for up to 3 equivalent months (720 hours, 8 hours/day) and immersed in water continuously for up to 3 weeks. The spectra were measured before and after the tests for comparison and the results are shown in Fig. 4b. The spectra of the emitter were almost identical after these tests, illustrating its long-term reliability and high resistance to UV irradiation and water. For comparison, we also conducted the same durability tests for a 100- $\mu \mathrm{m}$ PDMS film, which has been widely used in passive radiative coolers. After 400-hour UV exposure, a noticeable increase in the solar absorption of the PDMS film was observed (Supplementary Fig. S15).

\section{Conclusion}

In summary, we have developed an efficient all-inorganic narrowband emitter for daytime radiative cooling. It shows strong solar reflection $(96 \%)$ and highly selective IR emission $\left(\varepsilon_{8-13 \mu \mathrm{m}}=94.6 \%, \nabla_{\varepsilon}=1.44\right)$ within the atmospheric window, rendering it suitable for radiative cooling in hot humid climates. Its great IR-selective emission is realized by coupling the selective emission of PHPS-derived $\mathrm{SiO}_{\mathrm{x}} \mathrm{N}_{\mathrm{y}}$ with the narrowband polariton resonance of a monolayer of closely packed silica microspheres. Temperature drops of up to $5^{\circ} \mathrm{C}$ were achieved at noontime under high humidity with the emitter directly exposed to the ambient in a subtropical coastal city, Hong Kong. Furthermore, the high-performance emitter was fabricated by scalable solution-based fabrication processes at room temperature, rendering it promising for large-scale deployment. The excellent UV and water resistance of this inorganic emitter also enable its excellent durability in the harsh outdoor environment.

\section{Methods}

Sample preparation process. Commercial perhydropolysilazane (PHPS, 20 wt\%) was purchased from lota Silicone Oil (Anhui, China) company as a precursor for the silicon oxynitride layer. A layer of PHPS was deposited onto a silicon or metal substrate coated with 20nm titanium/120-nm silver by spray coating or spin coating, with thickness control through the concentration of PHPS and flow rate or rotational speed. The samples after the preliminary solidification in ambient air were then put into a plasma treatment system (March PX-250) to be exposed to $55 \mathrm{~W}$ oxygen plasma at 0.1 torr for 3 mins to complete surface hydrophilization, prepared for the next Langmuir-Blodgett (LB) process. An LB controlling system, including a surface pressure detector and a syringe pump from KSV NIMA, was combined with a customized enlarged LB trough to deposit a monolayer of silica microspheres on the silicon oxynitride. The hydrophobic silica microspheres were ordered from Tianjin Saierqun Technology Co. Ltd, which can float and evenly distribute on the water surface in the LB trough. Thereafter, a low-concentration PHPS (5 wt\%, diluted by N-tincyl ether) was sprayed or spin-coated onto the silica sphere monolayer to bond the silica sphere monolayer to the silicon oxynitride film. Finally, the samples were naturally solidified in the air or accelerated solidified by UV/solar irradiation at room temperature.

Characterizations. The morphologies of the fabricated samples were checked by Scanning Electron Microscopy (SEM, JSM-6390, JEOL) equipped with energy dispersive X-ray spectroscopy (EDX). The confirmation of composition from the cross-section of samples was carried out by the EDX method. The depth profiling analysis of the silicon oxynitride layer was conducted by X-ray Photoelectron Spectroscopy (XPS, PHI 5600 multi-technique system, Physical Electronics) in conjunction with ion beam sputtering and Time-of-Flight Secondary Ion Mass Spectrometry (ToF-SIMS, Physical Electronics 7200 ToF-SIMS spectrometer). For accurate characterization, a 
silicon wafer was used as the substrate and 20-nm titanium and 120-nm silver were deposited as adhesive and reflective layer respectively by e-beam evaporation (Peva-450E). The UV-visible-NIR $(0.3-2.5 \mu \mathrm{m})$ reflectance spectra of the emitters were measured using a spectrometer (Lambda 950, Perkin Elmer) equipped with a $150 \mathrm{~mm}$ integrating sphere. The mid-infrared emissive spectra were calculated from the reflectance spectra measured by Fourier transform infrared spectrometer (FTIR, Vertex 70, Bruker) with a gold-coated integrating sphere (PIKE Technologies). Angle adjustor was used for both spectrometers to measure angular reflection, from which angular emissive spectra can be gained. The water contact angles were measured by a contact angle meter (Biolin Theta).

Solar absorptance calculation. The spectrally averaged solar absorptance $\bar{\alpha}$ was defined as:

$$
\bar{\alpha}=\frac{{ }_{0.3 \mu m}^{3 \mu m} \alpha(\lambda) E_{\text {solar }}(\lambda) d \lambda}{I_{\text {solar }}}
$$

1

Here $E_{\text {solar }}(\lambda), a(\lambda)$ and $I_{\text {solar }}$ represent the spectral solar power (AM 1.5G), the spectral absorptance of surfaces at the wavelength $\lambda$, and total solar power,respectively.

Weighted emissivity and spectral effectiveness calculation. The weighted emissivity $\varepsilon_{8-13 \mu m}$ and the spectral effectiveness $\mathbb{Q}_{\varepsilon}$ were defined as:

$$
\varepsilon_{a-b \mu m}=\frac{{ }_{a \mu m}^{b \mu m} d \lambda I_{B B}\left(\lambda, T_{a m b}\right) \varepsilon(\lambda)}{{ }_{a \mu m}^{b \mu m} d \lambda I_{B B}\left(\lambda, T_{a m b}\right)}
$$

2

$$
\eta_{\varepsilon}=\frac{\varepsilon_{8-13 \mu m}}{\varepsilon_{0-\infty \mu m}}
$$

3

Here $I_{B B}$ and $\varepsilon(\lambda)$ represent the blackbody spectral radiance at the ambient temperature $T_{a m b}=300 \mathrm{~K}$ and spectral emissivity of the emitters.

Numerical simulations of the emitter. The spectrum simulations for the structures were performed using a commercial finite-difference time-domain (FDTD) method (Lumerical software).

Calculation of cooling performance. The cooling power was defined $\mathrm{as}^{4}$ :

$$
P_{\text {cool }}\left(T_{\text {emitter }}, T_{a m b}\right)=P_{\text {rad }}(T)-P_{a t m}\left(T_{a m b}\right)-P_{\text {solar }}-P_{\text {nonrad }}\left(T_{\text {emitter }}, T_{a m b}\right)
$$

3

where $P_{\text {rad }}$ is the radiative power from the emitter surface, $P_{\text {solar }}$ and $P_{a t m}$ are the absorbed radiation power from sunlight and atmosphere, respectively, and $P_{\text {nonrad }}$ is the nonradiative heat transfer rate. $T_{\text {emitter }}$ is the temperature of the emitter and $T_{a m b}$ is the temperature of ambient air. The maximum cooling power was attained when $T_{\text {emitter }}=T_{a m b}$. The maximum cooling temperature $\Delta T_{\text {max }}$ was defined as $\Delta T_{\max }=T_{a m b}-T_{\text {emitter }}$ when $P_{\text {cool }}=0$.

Cooling performance measurements. The ambient data including the ambient temperature, solar irradiation, relative humidity and wind speed were measured by a calibrated commercial portable weather station (Wuhan Yigu Chenyun Technology Co., Ltd, YG-BX). The emitters' temperatures were measured using T-type thermocouples connected to a high-density thermocouple module (NI 921316 TC). The cooling power was measured by maintaining the temperature of the emitter as the same as the temperature of ambient air through a heater combined with a Proportional-Integral-Derivative (PID) controller and recording the power input of the heater.

Ultraviolet resistance tests. The samples were put in a test box with two $40 \mathrm{~W}$ UV lamps installed inside. The irradiation of these UV lamps peaks at $340 \mathrm{~nm}$, which works as a solar ultraviolet simulator. The reflective spectra of samples were measured before and after the long-term (up to 720 hours) UV exposure.

Page 6/12 


\section{Declarations}

Data availability. The data sets generated and analysed during the current study are available from the corresponding author upon reasonable request.

\section{Acknowledgments}

This work was financially supported by the Sci. \& Tech. and Innovation Commission of Shenzhen Municipality of China (Project No. SGDX2019081623360564). We also thank the support from the Hong Kong Research Grant Council (RGC) via General Research Fund (GRF) account 16200518 and Collaborative Research Fund (CRF) account C6022-16G, respectively. The authors thank Prof. Ronggui Yang at Huazhong University of Science and Technology, China, for the helpful discussions.

\section{Author contributions}

C.L. and B.H. conceived and designed the research. C.L. and Y.L. performed the small samples characterization, optical simulation and measurements. C.L. and C.C. performed the hardware preparation and calibration. C.L. and Y.S.K. performed the big samples fabrication and filed tests. C.Y.T. and C.Y.H.C. provided many suggestions in the discussion of results and writing of the manuscript. C.L. and B.H., analyzed the results and wrote the manuscript. All authors approved the final version of the manuscript.

\section{Additional information}

Supplementary Information is available for this paper

Competing interests: The authors declare no competing interests.

\section{References}

1. Catalanotti, S. et al. The radiative cooling of selective surfaces. Sol. Energy 17, 83-89 (1975).

2. Trombe, F. Perspectives sur l'utilisation des rayonnements solaires et terrestres dans certaines regions du monde. (1975).

3. Raman, A. P., Anoma, M. A., Zhu, L., Rephaeli, E. \& Fan, S. Passive radiative cooling below ambient air temperature under direct sunlight. Nature 515, 540-544 (2014).

4. Liu, J. et al. Preliminary study of radiative cooling in cooling season of the humid coastal area. Sol. Energy Mater Sol. Cells 208, 110412 (2020).

5. Han, D., Ng, B. F. \& Wan, M. P. Preliminary study of passive radiative cooling under Singapore's tropical climate. Sol. Energy Mater Sol. Cells 206, 110270 (2020).

6. Khedari, J., Waewsak, J., Thepa, S. \& Hirunlabh, J. Field investigation of night radiation cooling under tropical climate. Renew. Energ. 20, 183-193 (2000).

7. Tso, C. Y., Chan, K. C. \& Chao, C. Y. A field investigation of passive radiative cooling under Hong Kong's climate. Renew. Energ. 106, 52-61 (2017).

8. Jeong, S. Y., Tso, C. Y., Wong, Y. M., Chao, C. Y. \& Huang, B. Daytime passive radiative cooling by ultra emissive bio-inspired polymeric surface. Sol. Energy Mater Sol. Cells 206, 110296 (2020).

9. Granqvist, C. G., Hjortsberg, A. \& Eriksson, T. S. Radiative cooling to low temperatures with selectivity IR-emitting surfaces. Thin Solid Films 90, 187-190 (1982).

10. Chen, Z., Zhu, L., Raman, A. \& Fan, S. Radiative cooling to deep sub-freezing temperatures through a 24-h day-night cycle. Nat. Commun. 7, 1-5 (2016).

11. Hossain, M. \& Gu, M. Radiative cooling: Principles, progress, and potentials. Adv. Sci. 3 (2016).

12. Zeyghami, M., Goswami, D. Y. \& Stefanakos, E. A review of clear sky radiative cooling developments and applications in renewable power systems and passive building cooling. Sol. Energy Mater Sol. Cells 178, 115-128 (2018).

13. Sun, X., Sun, Y., Zhou, Z., Alam, M. A. \& Bermel, P. Radiative sky cooling: fundamental physics, materials, structures, and applications. Nanophotonics 6, 997-1015 (2017).

14. Vall, S. \& Castell, A. Radiative cooling as low-grade energy source: A literature review. Renew. Sust. Energ. Rev. 77, 803-820 (2017). 
15. Zhao, B., Hu, M., Ao, X., Chen, N. \& Pei, G. Radiative cooling: A review of fundamentals, materials, applications, and prospects. Appl. Energy 236, 489-513 (2019).

16. Chae, D. et al. Spectrally selective inorganic-based multilayer emitter for daytime radiative cooling. ACS Appl. Mater. Interfaces 12, 8073-8081 (2020).

17. Zhai, Y. et al. Scalable-manufactured randomized glass-polymer hybrid metamaterial for daytime radiative cooling. Science $\mathbf{3 5 5}$, 1062-1066 (2017).

18. Atiganyanun, S. et al. Effective radiative cooling by paint-format microsphere-based photonic random media. ACS Photonics $\mathbf{5}$, 1181-1187 (2018).

19. Bao, H. et al. Double-layer nanoparticle-based coatings for efficient terrestrial radiative cooling. Sol. Energy Mater Sol. Cells 168, 7884 (2017).

20. Mandal, J. et al. Hierarchically porous polymer coatings for highly efficient passive daytime radiative cooling. Science 362 , 315-319 (2018).

21. Li, T. et al. A radiative cooling structural material. Science $364,760-763$ (2019).

22. Zweifel, H. in Stabilization of polymeric materials (Springer Science \& Business Media, 2012).

23. Wang, K., Günthner, M., Motz, G., Flinn, B. D. \& Bordia, R. K. Control of surface energy of silicon oxynitride films. Langmuir 29, 28892896 (2013).

24. Bardosova, M., Pemble, M. E., Povey, I. M. \& Tredgold, R. H. The langmuir-blodgett approach to making colloidal photonic crystals from silica spheres. Adv. Mater. 22, 3104-3124 (2010).

25. Greffet, J. et al. Coherent emission of light by thermal sources. Nature 416, 61-64 (2002).

26. Jaramillo-Fernandez, J. et al. A Self-Assembled 2D Thermofunctional Material for Radiative Cooling. Small, 1905290 (2019).

27. https://.

28. Lakatos, Á \& Kalmár, F. Investigation of thickness and density dependence of thermal conductivity of expanded polystyrene insulation materials. Mater. Struct. 46, 1101-1105 (2013).

29. Zhao, D. et al. Radiative sky cooling: Fundamental principles, materials, and applications. Appl. Phys. Rev. 6, 021306 (2019).

30. Granqvist, C. G. \& Hjortsberg, A. Radiative cooling to low temperatures: General considerations and application to selectively emitting SiO films. J. Appl. Phys. 52, 4205-4220 (1981).

31. Kou, J., Jurado, Z., Chen, Z., Fan, S. \& Minnich, A. J. Daytime radiative cooling using near-black infrared emitters. ACS Photonics 4, 626-630 (2017).

32. Lee, G. J., Kim, Y. J., Kim, H. M., Yoo, Y. J. \& Song, Y. M. Colored, daytime radiative coolers with thin-film resonators for aesthetic purposes. Adv. Opt. Mater. 6, 1800707 (2018).

33. Wang, X. et al. Scalable Flexible Hybrid Membranes with Photonic Structures for Daytime Radiative Cooling. Adv. Funct. Mater. 30, 1907562 (2020).

34. Aili, A. et al. Selection of polymers with functional groups for daytime radiative cooling. Mater. Today Phys. 10, 100127 (2019).

35. Zhang, H. et al. Biologically inspired flexible photonic films for efficient passive radiative cooling. Proc. Natl. Acad. Sci. U. S. A. 117, 14657-14666 (2020).

36. Rephaeli, E., Raman, A. \& Fan, S. Ultrabroadband photonic structures to achieve high-performance daytime radiative cooling. Nano Lett. 13, 1457-1461 (2013).

37. Zou, C. et al. Metal-Loaded Dielectric Resonator Metasurfaces for Radiative Cooling. Adv. Opt. Mater. 5, 1700460 (2017).

38. Li, D. et al. Scalable and hierarchically designed polymer film as a selective thermal emitter for high-performance all-day radiative cooling. Nat. Nanotechnol., 1-6 (2020).

\section{Figures}



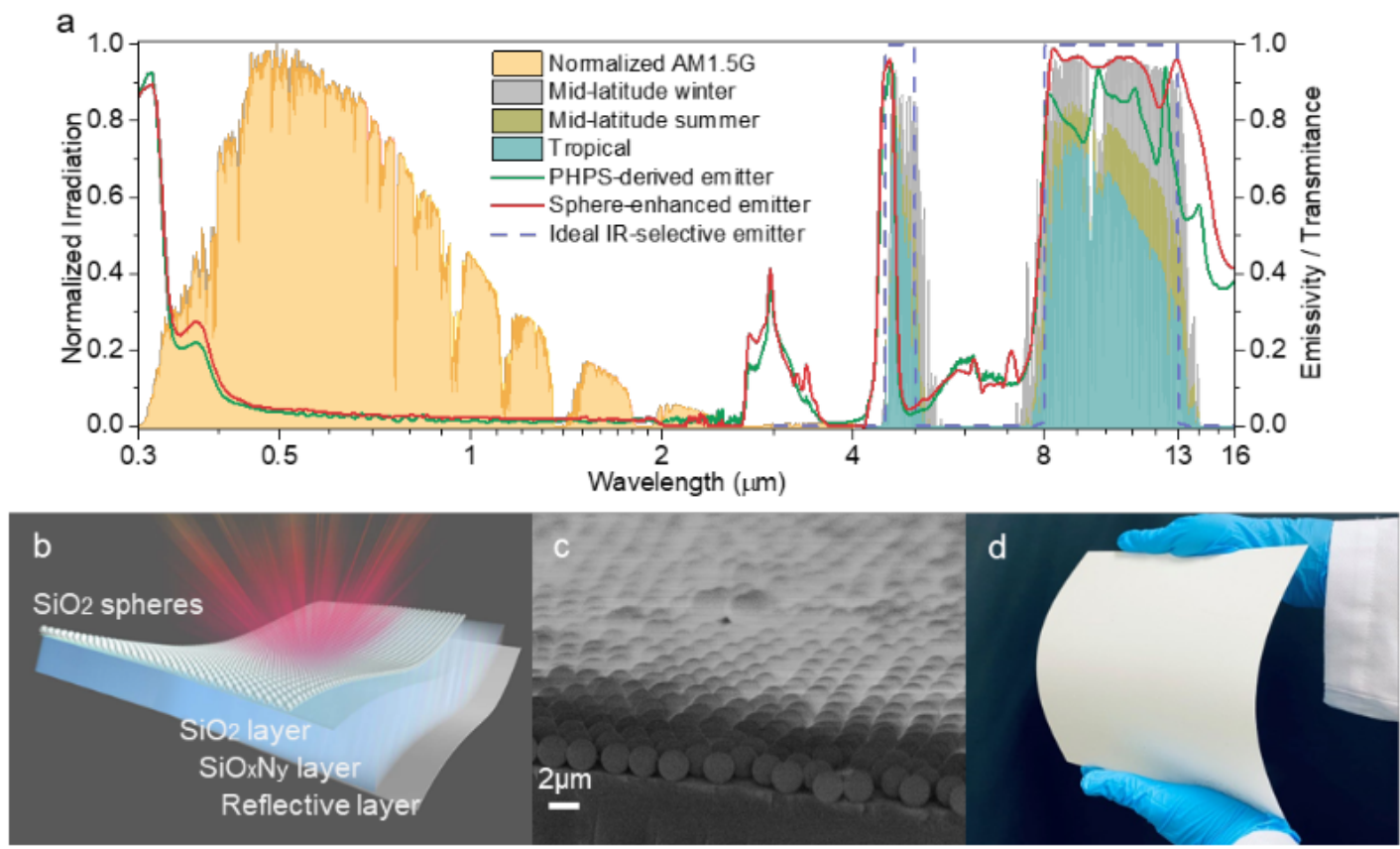

e PHPS deposition by spray coating

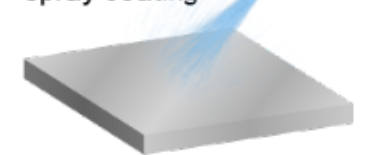

Final solidification by exposure of UV or Sunlight

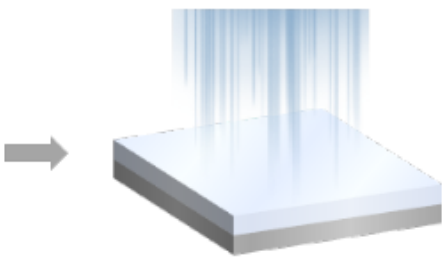

Preliminary solidification and surface hydrophilization by $\mathrm{O}_{2}$ plasma

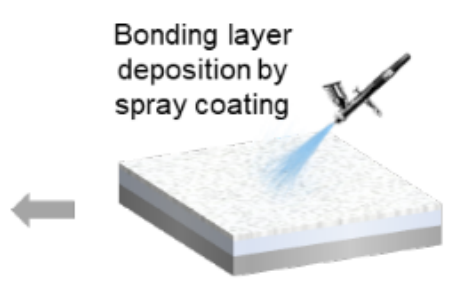

\section{Figure 1}

Spectra and fabrication processes of the inorganic emitter. a, Emissive spectra of the PHPS-derived and sphere-enhanced emitter. Normalized solar irradiation and atmospheric transmittances in different climates were plotted as references. b, Schematic, c, scanning electron micrograph and d, photograph of the emitter $(20 \times 20 \mathrm{~cm})$. e, Schematic of fabrication processes of the emitter. 
a

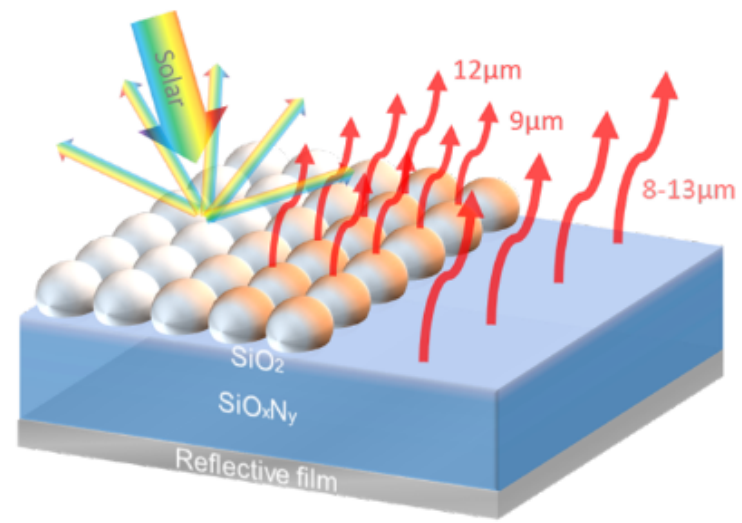

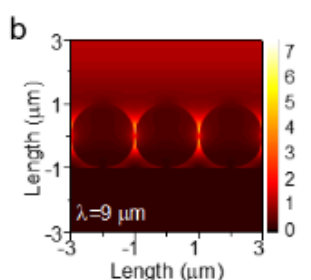

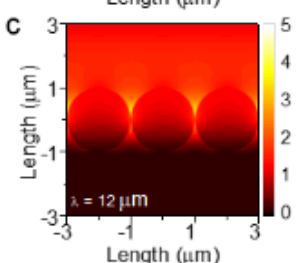

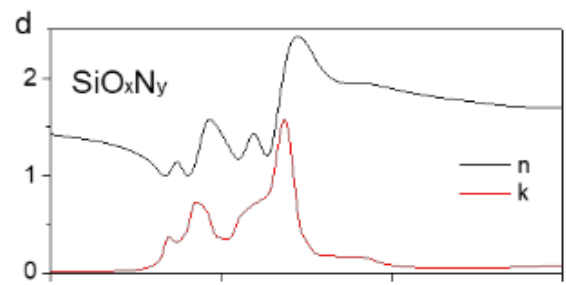

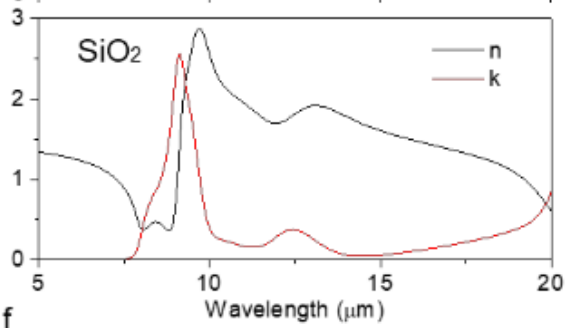

$f$

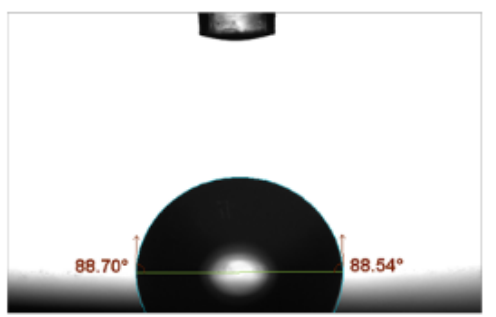

Without spheres

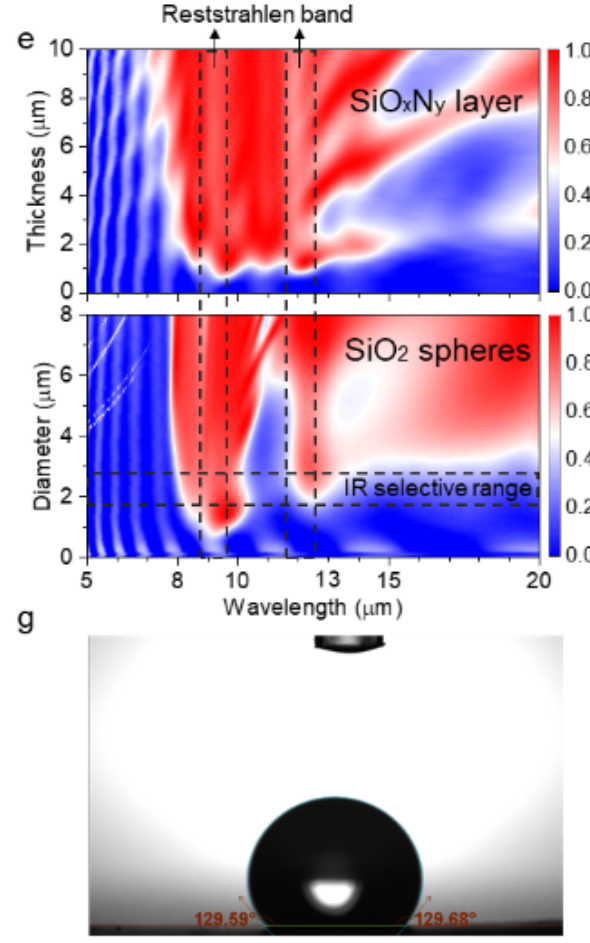

With spheres

\section{Figure 2}

Optical properties of the emitter. a, The optical structure of the emitter. b-c, Electric field enhancement at $9 \mu \mathrm{m}$ (b) and $12 \mu \mathrm{m}$ (c) by polariton resonance. $d$, Refractive index and extinction coefficient of bulk silicon oxynitride (upper panel) and silicon dioxide (lower panel) e, Calculated emissive spectra for silicon oxynitride (upper panel) and close-packed SiO2 sphere monolayer (lower panel) on a reflective surface as functions of the layer thickness and sphere size, respectively. $f-g$, The water contact angles of the PHPS-derived emitter without (f) and with $(\mathrm{g})$ the silica microsphere monolayer. 


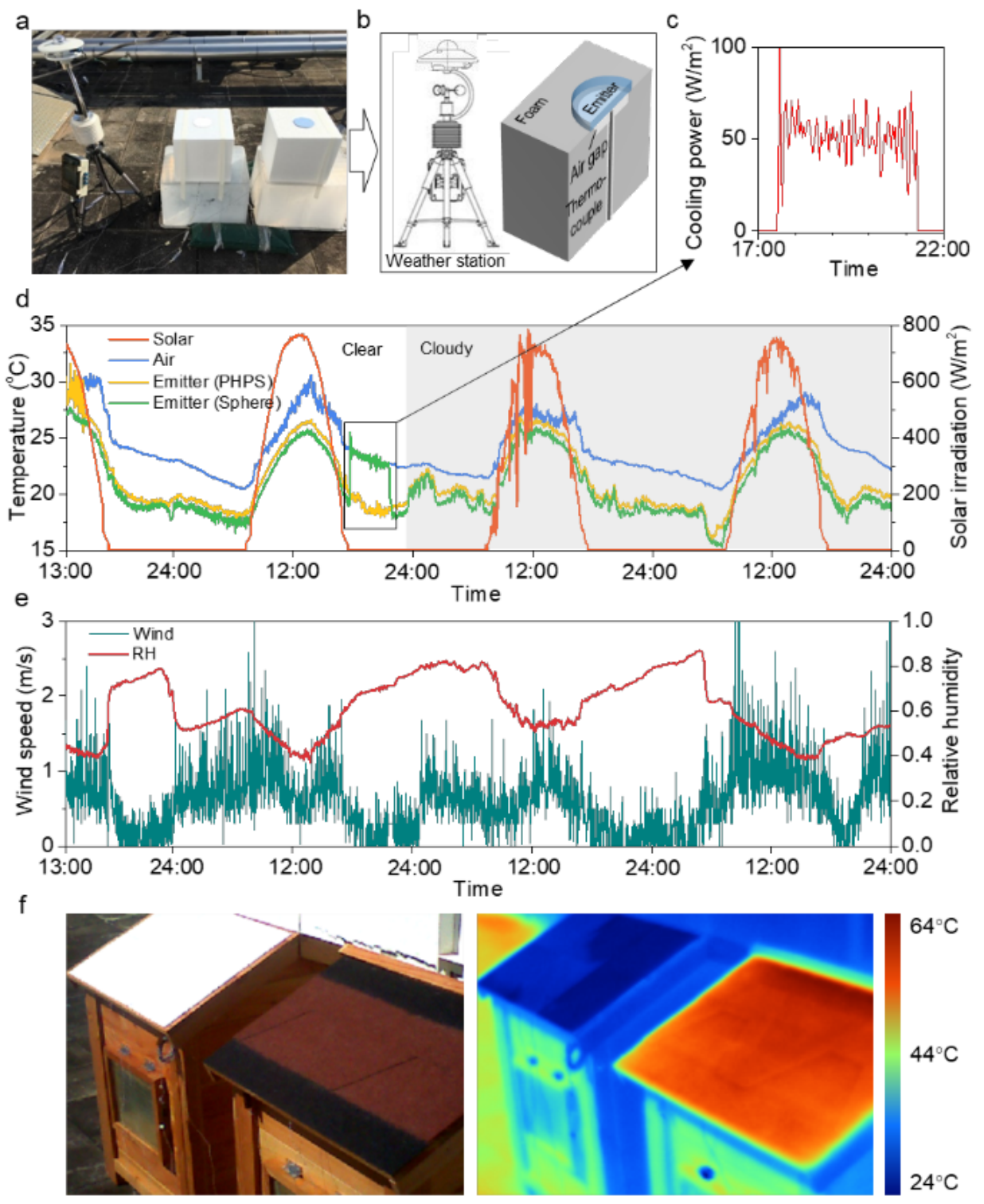

\section{Figure 3}

Outdoor experiment in a subtropical coastal city (Hong Kong). a, Picture of the setup with sphere-enhanced emitter (left) and PHPSderived emitter (right). b, Schematic of the platform (b). c, Measured cooling power under a high humidity level ( $70 \%)$. d, Measured temperatures of the ambient air and the emitters (include PHPS-derived and sphere-enhanced emitters) and solar irradiation under clear and cloudy skies during the test. e, Continuous measurement of wind speed and relative humidity in the meantime. f, Visible (left) and IR (right) images of two wooden model houses $(60 \times 70 \times 85 \mathrm{~cm})$ with a standard thermal insulating roof and an emitter-assembled roof under direct sunlight at noontime when the ambient air temperature was $\sim 31{ }^{\circ} \mathrm{C}$. 
a

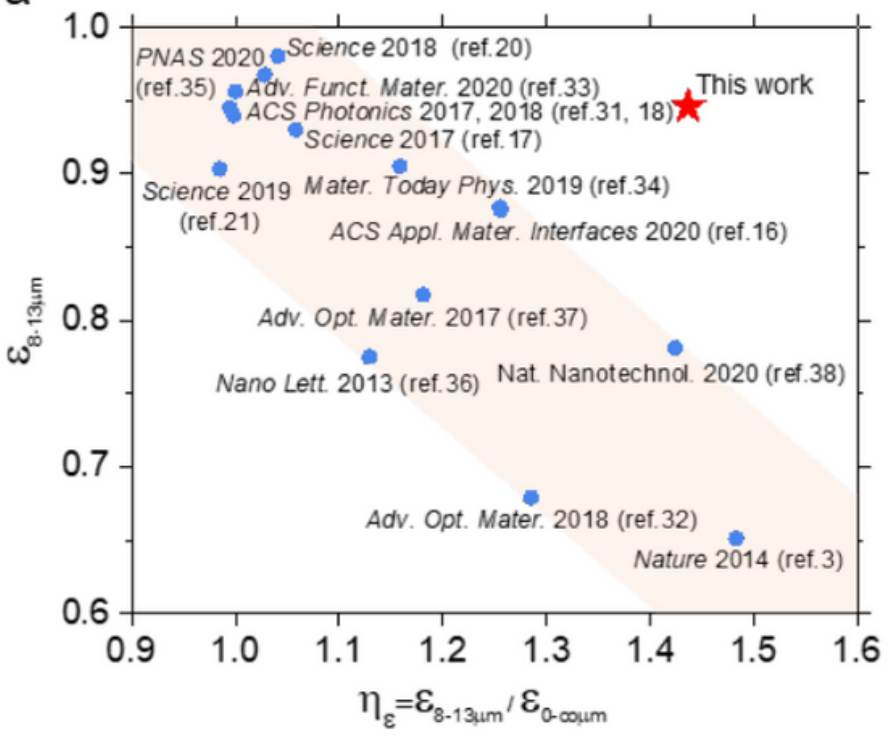

b

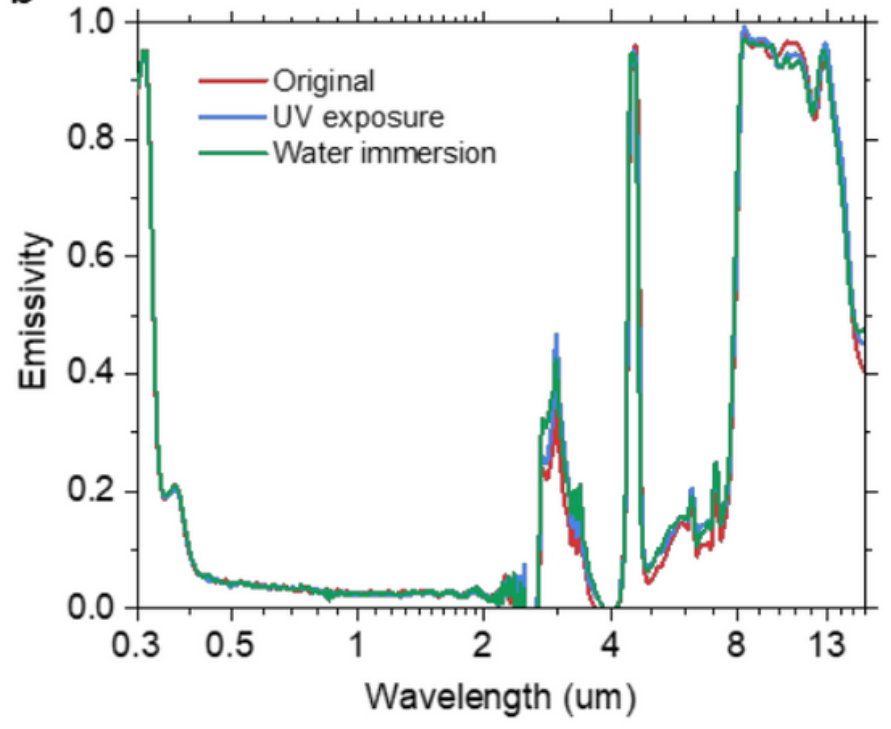

\section{Figure 4}

Cooling potential and reliability of emitters. a, Cooling potentials $(\varepsilon 8-13 \mu \mathrm{m}$ for cooling power and $\llbracket \varepsilon$ for cooling temperature) of recently reported high-performance emitters for daytime radiative cooling and the emitter in this work. b, Spectra of the sphere-enhanced emitter before and after long-term solar UV exposure (3 months) and water immersion (3 weeks).

\section{Supplementary Files}

This is a list of supplementary files associated with this preprint. Click to download.

- SIASolutionprocessedInorganicEmitterwithHighSpectralEffectivenessforEfficientDaytimeRadiativeCoolinginHotHumidClimates.docx 